Pecvnia, 6 (2008), pp. 153-174

\title{
¿Financian los franquiciados la expansión de los franquiciadores?: Evidencia para el caso español
}

Recibido: Abril 2008

Aceptado: Junio 2008
Vanesa Solís Rodríguez

vanesa.solis@unileon.es

Universidad de León

Fac. de Ciencias Económicas y Empresariales Campus de Vegazana, s/n

24071 León (España)

Manuel González Díaz

mgdiaz@uniovi.es

Universidad de Oviedo

Fac. de Ciencias Económicas y Empresariales

Avenida del Cristo, $\mathrm{s} / \mathrm{n}$

33071 Oviedo (España)
Este trabajo tiene como objetivo analizar si la dificultad para acceder a los recursos financieros es un factor determinante de la propensión franquiciadora de las cadenas españolas. Para ello, se ha tomado como muestra un panel de datos formado por cadenas observadas entre 1996 y 2002. Además, empleamos un modelo dinámico de ajuste parcial de la estructura de propiedad de las cadenas utilizando el método generalizado de los momentos en primeras diferencias, lo que nos permite, no sólo introducir un componente autorregresivo, sino también el empleo de variables explicativas endógenas. Los
This work considers whether the difficulty for obtaining funding affects the choice of organizational form for franchise chains. We created a panel of Spanish franchisers who were observed from 1996 to 2002, and calculated a dynamic, partial-fit model for ownership structure, using the generalised method of moments in first differences. This allowed us to introduce auto-regression and to use endogenous explanatory variables. The results obtained show that the greater a chain's liquidity and profitability and the lower its debt, the less likely it is to operate as a franchiser. This supports the financial argument. 
resultados obtenidos muestran que cuanto mayor es la liquidez y la rentabilidad económica y menor es el endeudamiento de una determinada cadena menor es su propensión franquiciadora, lo que apoya el argumento financiero. Por el contrario, los requisitos de inversión necesarios para la expansión no son significativos. Además, la antigüedad presenta un signo contrario al previsto. Finalmente, observamos que se verifica el modelo de ajuste parcial de la estructura de propiedad, obteniendo una elevada velocidad de ajuste, entre un $78-69 \%$.

Palabras clave: Franquicia; Escasez de capital; Datos de panel; Evolución.

\begin{abstract}
However, the requirements for investment in growth are not significant, and age shows does not show the expected sign. Finally, we verified the partial-fit model for ownership structure, obtaining a high adjustment speed of $78-69 \%$.
\end{abstract}

Key words: Franchising; Capital scarcity; Panel data; Evolution.

\section{INTRODUCCIÓN}

Aunque la literatura sobre franquicias es extensa ${ }^{1}$, una cuestión que está pendiente de resolver es por qué los estudios empíricos no respaldan la opinión de los empresarios (franquiciadores) respecto al interés de esta fórmula empresarial como medio de financiación (Dant 1995). Así, la mayor parte de los trabajos encuentran respaldo a la explicación teórica más aceptada que establece que las cadenas se deciden a franquiciar porque al convertir al responsable (o gerente) de un establecimiento en un "empresario" retribuido con la renta residual se alinean mejor sus intereses con los de la cadena, atenuando los problemas de control e incentivos que suelen surgir en esta relación ${ }^{2}$. Existe, sin embargo, otro argumento teórico que establece que la franquicia se utiliza para facilitar el acceso a determinados recursos escasos ${ }^{3}$. Así, las empresas franquician parte de sus establecimientos cuando tienen dificultades para acceder a determinados recursos escasos, tales como el capital (Ozanne y Hunt 1971; Caves y Murphy 1976), las habilidades directivas (Oxenfeldt y Kelly 1969; Norton 1988) y la información local (Minkler 1990). Una vez que hayan conseguido el acceso a dichos recursos,

\footnotetext{
Véase, a modo de recopilación de la investigación efectuada durante más de 25 años sobre la franquicia, el reciente trabajo de Blair y Lafontaine (2005).

2 Véase Caves y Murphy (1976) y, sobre todo, Rubin (1978).

3 Los primeros en establecer este argumento fueron Oxenfeldt y Kelly (1969).
} 
las cadenas se dedicarán a recomprar los establecimientos franquiciados, aspirando a tener la propiedad de todos sus puntos de venta. A diferencia del primer argumento, este segundo ha generado, y sigue generando, un gran debate, puesto que los resultados obtenidos son dispares.

Por ello, nuestro objetivo es analizar si la dificultad para acceder a determinados recursos escasos (financieros) es un factor determinante de la propensión franquiciadora de las cadenas españolas. La mayor parte de los estudios realizados toman como muestra cadenas norteamericanas, cuyo mercado de capitales no es comparable al español. Es, por tanto, interesante determinar si la falta de relevancia del argumento financiero se mantiene para el caso español o si, por el contrario, existen diferencias. Por otra parte, la mayor novedad de este artículo radica en otro aspecto, que es el empleo de datos de panel. Hasta la fecha, muy pocos son los trabajos que han tratado, directa o indirectamente, de contrastar el argumento financiero en España, siendo todos ellos de corte transversal ${ }^{4}$. A nivel internacional, solamente el artículo de Minkler y Park (1994), que toma como muestra cadenas norteamericanas, incorpora esta metodología para tratar de contrastar el argumento financiero ${ }^{5}$. La ventaja de la misma radica en que nos permite controlar la heterogeneidad inobservable presente en las diferentes cadenas que componen la muestra. Dicho de otra manera, nos permite estimar los efectos individuales de cada cadena, cuestión que no es recogida con las regresiones de corte transversal. Además, en este caso utilizamos un modelo dinámico de ajuste parcial de la estructura de propiedad de las cadenas, utilizando el método generalizado de los momentos en primeras diferencias. Esto no sólo permite introducir un componente autorregresivo en el modelo, fundamental para aumentar su capacidad explicativa, sino también utilizar variables explicativas endógenas.

El resto del artículo se organiza como sigue. En la segunda sección efectuamos una revisión teórica del por qué aparecen las franquicias, centrándonos en el argumento de escasez de recursos financieros. En la tercera sección se describe el proceso de obtención de datos, las fuentes de información utilizadas y el modelo econométrico

\footnotetext{
Algunos ejemplos son los trabajos de López y Ventura $(2001,2002)$.

Los trabajos de Pénard, Raynaud y Saussier (2003), que toma como muestra cadenas francesas, y de Lafontaine y Shaw (2005), centrado en el mercado estadounidense, al igual que el de Minkler y Park, también emplean datos de panel, pero para contrastar argumentos diferentes al de escasez de recursos financieros.
} 
empleado. Finalmente, los resultados y conclusiones del trabajo son recogidas, respectivamente, en las secciones cuarta y quinta.

\section{REVISIÓN TEÓRICA}

La franquicia es una forma organizativa híbrida por la cual una empresa, la franquiciadora, cede a otra, la franquiciada, el derecho a utilizar una marca o fórmula comercial de reconocido prestigio en unas determinadas condiciones y a cambio de una contraprestación financiera ${ }^{6}$.

La explicación más aceptada sobre su aparición es que soluciona el problema de agencia entre la cadena y los responsables de cada establecimiento. Este problema consiste en que cuando propietario y directivo no coinciden en la misma persona, el último puede perseguir sus propios intereses en lugar de los del principal a quien representa (escaqueo en el esfuerzo, emplear el coche de la compañía para uso particular, etc.). El motivo de este comportamiento es que no carga con todos los costes derivados del mismo, especialmente cuando su salario es fijo. Por tanto, se generará un coste de agencia como consecuencia de los conflictos de incentivos existentes entre esos directivos y la propia compañía (Rubin 1978; Brickley, Dark y Weisbach 1991). Al convertir al responsable de un establecimiento en franquiciado, otorgándole los derechos residuales de su establecimiento (por ejemplo, la renta residual), los intereses de las partes quedan mucho mejor alineados.

La evidencia empírica a favor de este argumento es prácticamente unánime. Ahora bien, en base al mismo, se debería concluir que lo mejor que pueden hacer las cadenas es franquiciar el mayor número de establecimientos posible, puesto que, de esa manera, menores serán los problemas de control y motivación de los responsables de los establecimientos y, por tanto, más eficiente será la organización.

Sin embargo, en la práctica, lo que se observa es que las cadenas de franquicia combinan el uso de establecimientos propios y franquiciados, lo que se ha denominado forma plural (Bradach y Eccles 1989; Bradach 1997), que consiste en el mantenimiento simultáneo de 
establecimientos propios, gobernados jerárquica o empresarialmente, y franquiciados, gobernados a través de acuerdos en el mercado ${ }^{7}$. Por lo tanto, es lógico pensar que existan otros argumentos que, junto con el argumento de agencia, influyan en la decisión a franquiciar.

Uno de estos argumentos es el de escasez de recursos, desarrollado inicialmente por Oxenfeldt y Kelly (1969). Estos autores establecen que el deseo de todo franquiciador es que todos sus establecimientos estén bajo su propiedad. Sin embargo, se decidirá a franquiciar parte de los mismos cuando tenga dificultades para acceder a determinados recursos escasos. Una vez que haya conseguido el acceso a los mismos, se dedicará a recomprar los establecimientos franquiciados, aspirando a tener, como se señaló anteriormente, la propiedad de todos sus puntos de venta. Por lo tanto, para estos autores, la utilidad de la franquicia para el franquiciador cambia con el paso del tiempo: "...la franquicia es útil para un franquiciador exitoso principalmente durante la infancia y la adolescencia de la empresa..." (Oxenfeldt y Kelly 1969: 69).

Estos recursos escasos pueden ser, fundamentalmente, de tres tipos: capital (Ozanne y Hunt 1971; Caves y Murphy 1976), habilidades directivas (Oxenfeldt y Kelly 1969; Norton 1988) e información local (Minkler 1990). En este trabajo nos centramos en el primero de ellos, el cual pasamos a describir a continuación.

\subsection{Argumento de escasez de recursos financieros}

La escasez de capital es la restricción de recursos más obvia a la que se enfrentan las empresas para conseguir crecer (López y Ventura 2002). En sus etapas iniciales de vida, las empresas tienen dificultades para acceder a la financiación necesaria para el crecimiento de su red comercial $^{8}$, de tal manera que recurren a franquiciar parte de sus establecimientos para que los franquiciados les proporcionen ese capital que de otra manera les resultaría inaccesible. De esta manera, el franquiciador consigue los recursos financieros necesarios para poder ampliar su negocio

\footnotetext{
7 Véase Williamson (1991). Nos referimos en este caso a los distintos mechanisms of governance, de ahí que hayamos utilizado el término gobernado, en el sentido de estructurado u organizado.

8 Nos referimos tanto a que tienen dificultades para acceder al mercado de capitales como a que no tienen capacidad interna para asumir la expansión del negocio.
} 
(Ozanne y Hunt 1971; Caves y Murphy 1976) y, posteriormente, a medida que la compañía madure, se dedicará a recomprar esos establecimientos que anteriormente había franquiciado (Oxenfeldt y Kelly 1969; Hunt 1973) ${ }^{9}$.

Desde sus inicios, la idea de que la escasez de recursos financieros pudiera influir en la decisión de franquiciar de una determinada compañía ha generado grandes críticas. La más importante de todas ha sido la de Rubin (1978). Este autor afirma que este argumento va en contra de la teoría moderna de capitales al plantarse la siguiente cuestión: ¿Qué tiene mayor riesgo para un individuo: convertirse en franquiciado o en accionista de una franquicia? En principio, parece lógico pensar que lo primero. Existe más inseguridad y, por tanto, es más arriesgado para el franquiciado invertir en un único negocio que comprar acciones de la franquicia ${ }^{10}$. Por tanto, al estar asumiendo un mayor riesgo, también exigirá una compensación mayor. En consecuencia, es cierto que los franquiciados aportan capitales al franquiciador, pero estos capitales son costosos. $\mathrm{Si}$ verdaderamente la franquicia surgiese como consecuencia de las dificultades financieras, lo más eficiente para el franquiciador sería poner a la venta acciones de su cadena porque, de esta manera, obtendría un capital más barato. Además, de esta manera, le resultaría más sencillo encontrar individuos dispuestos a invertir en la compañía, al no tener éstos que implicarse directamente en la gestión del negocio (Rubin 1978) ${ }^{11}$.

Junto con la crítica anterior, ha sido bastante numerosa la evidencia empírica en contra de este argumento. En primer lugar, si, como se ha señalado, los empresarios recurren a la franquicia por tener problemas de financiación, cabría esperar que fueran las pequeñas empresas las que recurrieran a esta fórmula, puesto que son las que más dificultades tienen a la hora de acceder al mercado de capitales. Sin embargo, en la práctica, nos encontramos con que las grandes compañías también utilizan este sistema, a pesar de que no tienen ningún problema de acceso a dicho mercado (Brickley, Dark y Weisbach 1991) ${ }^{12}$. Por otra parte, también se

9 Las únicas franquicias que se mantendrán, si es que se mantiene alguna, serán las menos rentables y/o las situadas en las zonas rurales (Oxenfeldt y Kelly 1969).

10 En este segundo caso, está diversificando su cartera.

11 Posteriormente, Lafontaine (1992) examina este argumento, llegando a la conclusión de que sólo sería correcto si no se tienen en cuenta en el análisis los problemas de agencia. Otros autores, como Martin y Justis (1993), Shane (1996) o Bradach (1997) también llegan a esta conclusión.

12 Un ejemplo significativo es el de McDonalds o, en España, Telepizza. 
ha observado que cuanto mayor es la inversión unitaria requerida por parte de un franquiciado para poder abrir un establecimiento de una determinada cadena menos recurren los empresarios a la franquicia, lo que va en contra de este argumento (Brickley y Dark 1987; Brickley, Dark y Weisbach 1991; Lafontaine 1992; López y Ventura 2002). Por último, en muchas ocasiones, el propio franquiciador ayuda al franquiciado a obtener la financiación que necesita para poner en marcha su negocio, ya sea dándosela él mismo o ayudándole a negociar con las entidades financieras, las cuales suelen conceder créditos con intereses favorables a los negocios de franquicia que se abren.

No obstante, y a pesar de las críticas anteriores, la evidencia empírica a favor del argumento financiero es también muy numerosa. Por una parte, hay estudios que han contrastado esta hipótesis de una manera directa, ya sea empleando proxies de la escasez de capital o preguntándoles directamente a los franquiciadores por qué se deciden a franquiciar. Así, en el primer caso, Caves y Murphy (1976), Lafontaine y Kaufmann (1994), Combs y Castrogiovanni (1994) y Dant y Kaufmann (2003) observan una mayor proporción de establecimientos propios en aquellas cadenas de franquicia más maduras. Otros autores, como Thompson (1992) o Martin y Justis (1993), obtienen evidencia a favor de un mayor uso de la franquicia cuanto mayor es la inversión inicial necesaria para la apertura de un nuevo establecimiento. Por su parte, Dant y Kaufmann (2003) y Castrogiovanni, Combs y Justis (2006) obtienen una relación negativa entre el porcentaje de establecimientos franquiciados y el tamaño de la cadena, la cual apoya este argumento. Finalmente, autores como Norton (1995) y Combs y Ketchen (1999) han empleado medidas más específicas de la escasez de capital, como el ratio de endeudamiento o de liquidez, obteniendo también unos resultados favorables ${ }^{13}$. En el segundo caso, cuando a los propios franquiciadores se les pregunta por qué acuden a la franquicia responden que "...la razón más importante para adoptar el sistema de franquicia es la de conservar o adquirir capital, al mismo tiempo que se intenta establecer una red de distribución efectiva en el menor tiempo posible" (McGuire 1971: 6). Otros autores han obtenido resultados similares. Así, por ejemplo, Lafontaine (1992) encontró que 76 de los 130 franquiciadores encuestados para su estudio veían a la franquicia como un

13 Las variables de tamaño y antigüedad de la cadena pueden medir, no sólo la escasez de recursos financieros, sino también de habilidades directivas e información local, con lo que sus resultados pueden ser más ambiguos. 
mecanismo para proveerse de los fondos necesarios para la rápida expansión de su negocio. Dant (1995) también observó que la falta de capital era una de las principales razones por la que las empresas comenzaban a franquiciar, al obtener que el $60 \%$ de los directivos encuestados señalaban el acceso al capital como la razón por la que utilizaban el sistema de franquicia.

Otros estudios señalan indirectamente el valor de la hipótesis de la escasez de capital a la hora de explicar el empleo de la franquicia (Combs y Ketchen 1999). Así, Martin y Justis (1993) comparan el crecimiento de las cadenas de franquicia maduras e inmaduras ${ }^{14}$, llegando a la conclusión de que son las segundas las que dependen en mayor medida de la franquicia en las épocas de difícil acceso al mercado de capitales. Esta misma variable ha sido empleada por otros autores como Thompson (1992) o Combs y Castrogiovanni (1994). Por su parte, Sen (1993) observa que las cadenas más jóvenes establecen a sus franquiciados cánones de entrada más elevados que los establecidos por las cadenas maduras, lo que evidencia sus mayores dificultades para acceder al mercado de capitales. Por último, Michael (2002) demuestra la existencia en el mercado de las "ventajas de mover primero" en el sector de la restauración, lo que hace que los empresarios tengan incentivos a franquiciar con el objetivo de tener una ventaja competitiva sobre el resto de empresas del sector ${ }^{15}$. La Tabla 1 nos muestra parte de los estudios que han analizado este argumento.

En definitiva, si el argumento establecido es correcto, debe esperarse que medidas de la escasez de capital por parte de la empresa se encuentren asociadas con una mayor propensión a franquiciar los puntos de venta. Por tanto, la hipótesis a contrastar en este trabajo es la siguiente:

$\mathrm{H}_{1}$ : cuanto más difícil resulte para una determinada compañía el acceso a los recursos financieros mayor será su propensión a franquiciar.

14 Estos autores establecen que son cadenas de franquicia inmaduras aquellas que llevan operando en el mercado menos de 10 años y su número total de establecimientos es menor o igual al número medio de establecimientos en el sector.

15 Este autor demuestra con este resultado que la franquicia es un mecanismo para la adquisición de recursos puesto que la existencia de ventajas de mover primero es una de las presunciones claves del argumento de escasez de recursos. Así, "Inicialmente, muchos franquiciadores establecen franquicias con el objetivo de penetrar en el mercado lo más rápido posible, obteniendo, de esa manera, territorio valioso de los competidores" (Oxenfeldt y Kelly 1969: 74). 
Tabla 1: Efecto de la escasez de recursos financieros sobre la propensión a franquiciar

\begin{tabular}{|c|c|c|c|c|}
\hline Autor/es & Año & Datos & Medida & $\begin{array}{c}\% \text { de } \\
\text { franquiciados }\end{array}$ \\
\hline Caves y Murphy & 1976 & Datos sectoriales & Antigüedad & $(-)$ \\
\hline Brickley y Dark & 1987 & $\begin{array}{l}\text { 1. Empresas de diversos sectores } \\
\text { 2. Establecimientos de } 36 \text { cadenas } \\
\text { de diversos sectores }\end{array}$ & $\begin{array}{l}\text { 1. Inversión inicial } \\
\text { 2. Inversión inicial }\end{array}$ & - \\
\hline $\begin{array}{l}\text { Brickley, Dark y } \\
\text { Weisbach }\end{array}$ & 1991 & $\begin{array}{l}\text { 1. Datos sectoriales a nivel estatal } \\
\text { 2. Datos de establecimientos de } 36 \\
\text { cadenas }\end{array}$ & $\begin{array}{l}\text { 1. Tamaño } \\
\text { 2. Inversión inicial }\end{array}$ & $\begin{array}{l}(+) \\
-\end{array}$ \\
\hline Thompson & 1992 & Franquicias de diversos sectores & $\begin{array}{l}\text { 1. Inversión inicial } \\
\text { 2. Crecimiento }\end{array}$ & $\begin{array}{l}+ \\
+\end{array}$ \\
\hline Lafontaine & 1992 & $\begin{array}{l}\text { Franquicias de negocio de todos } \\
\text { los sectores }\end{array}$ & Inversión inicial & - \\
\hline Martin y Justis & 1993 & $\begin{array}{l}57 \text { cadenas de franquicia de los } \\
\text { sectores de fast food, hoteles y } \\
\text { tiendas de conveniencia }\end{array}$ & $\begin{array}{l}\text { 1. Inversión inicial } \\
\text { 2. Crecimiento }\end{array}$ & $\begin{array}{l}+ \\
+\end{array}$ \\
\hline Lafontaine y Kaufmann & 1994 & $\begin{array}{l}130 \text { encuestas a presidentes de los } \\
\text { sistemas de franquicia de EE.UU. }\end{array}$ & $\begin{array}{l}\text { 1. Antigüedad } \\
\text { 2. Variable dummy con valor } \\
1 \text { si el franquiciador es una } \\
\text { filial de una compañía más } \\
\text { grande }\end{array}$ & $\begin{array}{l}(-) \\
(-)\end{array}$ \\
\hline Minkler y Park & 1994 & Panel de datos 1975-1989 & $\begin{array}{l}\text { 1. Ratio de interés real } \\
\text { 2. Crecimiento } \\
\text { 3. Antigüedad }\end{array}$ & $\begin{array}{c}- \\
- \\
(+)\end{array}$ \\
\hline Combs y Castrogiovanni & 1994 & 558 cadenas de franquicia & $\begin{array}{l}\text { 1. Tamaño } \\
\text { 2. Antigüedad } \\
\text { 3. Crecimiento }\end{array}$ & $\begin{array}{l}+) \\
(-) \\
+\end{array}$ \\
\hline Norton & 1995 & $\begin{array}{l}25 \text { cadenas de franquicia y } 25 \text { no } \\
\text { franquicias }\end{array}$ & $\begin{array}{l}\text { Ratio de endeudamiento en } \\
\text { libros y de mercado }\end{array}$ & + \\
\hline Combs y Ketchen & 1999 & 91 cadenas de restaurantes & $\begin{array}{l}\text { 1. Pert } \\
\text { 2. Ratio de endeudamiento } \\
\text { 3. Liquidez } \\
\text { 4. Rentabilidad económica }\end{array}$ & $\begin{array}{l}(-) \\
+ \\
(-) \\
(-)\end{array}$ \\
\hline López y Ventura & 2002 & $\begin{array}{l}270 \text { centrales de franquicia } \\
\text { operantes en España }\end{array}$ & $\begin{array}{l}\text { 1. Inversión inicial } \\
\text { 2. Antigüedad } \\
\text { 3. Crecimiento }\end{array}$ & $\begin{array}{l}- \\
(-) \\
-\end{array}$ \\
\hline Dant y Kaufmann & 2003 & $\begin{array}{l}152 \text { encuestas a cadenas de fast } \\
\text { food }\end{array}$ & $\begin{array}{l}\text { 1. Tamaño } \\
\text { 2. Antigüedad } \\
\text { 3. Acceso interno a recursos } \\
\text { financieros }\end{array}$ & $\begin{array}{l}(-) \\
(-) \\
(-)\end{array}$ \\
\hline $\begin{array}{l}\text { Castrogiovanni, Combs y } \\
\text { Justis }\end{array}$ & 2006 & 439 cadenas de franquicia & $\begin{array}{l}\text { 1. Tamaño } \\
\text { 2. Antigüedad }\end{array}$ & $\begin{array}{l}(-) \\
(+)\end{array}$ \\
\hline
\end{tabular}

Nota: Los paréntesis de la última columna indican que la variable empleada para estimar la dificultad de acceso a los recursos financieros se encuentra inversamente relacionada con la magnitud de tales recursos financieros, de tal manera que es de esperar que su signo sea el opuesto al esperado.

Fuente: Elaboración propia. 


\section{METODOLOGÍA}

\subsection{Obtención de datos}

La hipótesis anterior ha sido contrastada utilizando un panel de datos, en el cual se recoge la información relativa a cadenas de franquicia españolas que se encontraban operando en nuestro país dentro del periodo 1996-2002. Los datos empleados fueron obtenidos, fundamentalmente, de las Guías Profesionales de la Franquicia editadas por Tormo\&Asociados, punto de partida de la base de datos, y Barbadillo\&Asociados, del Anuario Español del Franchising, editado por Franchisa, y del Libro Oficial de la Franquicia en España, editado por la Asociación Española de Franquiciadores. Para ello, se partió de las guías que recogían los datos relativos a 1996 y se fueron añadiendo todas las cadenas que iban apareciendo cada año.

Toda esta información se completó con la obtenida de páginas Web de los franquiciadores, memorias anuales y noticias de prensa como consecuencia de tres problemas. Primero, la falta de congruencia que fue encontrada para algunos datos al comparar la información de las diferentes guías. Segundo, a la hora de ir añadiendo los datos de las cadenas para los diferentes años, se encontraron varias cadenas que cambiaban de nombre de un año para otro. Esto planteó dudas sobre si se trataba de la misma cadena o si era una nueva con un nombre parecido a la ya existente. Caso a caso se fueron resolviendo esas dudas con toda la información que se pudo encontrar. Por último, señalar que fueron eliminadas de la base cadenas que las guías indicaban que eran españolas y que, en realidad, no lo eran.

Por su parte, la información de tipo financiero fue obtenida de la base de datos SABI, la cual provee información financiera sobre todas las empresas operantes en España. Al final, y una vez depurada toda la información disponible en la base, la muestra definitiva quedó integrada por 1.229 cadenas. No obstante, la falta de información sobre algunas variables obligó a utilizar muchas menos cadenas en las regresiones.

\subsection{Descripción del modelo y variables}

El modelo empírico se ha elegido teniendo en cuenta la potencial naturaleza dinámica de la estructura de propiedad en las cadenas de franquicia, sugerida en los resultados obtenidos por Pénard, Raynaud y Saussier (2003) y Lafontaine y Shaw (2005). En ambos trabajos se concluye 
gráficamente que las cadenas de franquicia parecen evolucionar hasta alcanzar una estructura de propiedad objetivo, momento a partir de la cual permanecen más o menos estables. Por lo tanto, las cadenas de franquicia reajustarán periódicamente su estructura hacia lo que consideran su proporción de establecimientos propios y franquiciados objetivo, de la cual no desean salir. Dado que en trabajos previos la estimación se ha hecho de forma gráfica, necesitamos analizar si verdaderamente existe dicho nivel objetivo y, si es así, cuál es la velocidad de ajuste con la que la empresa se mueve hacia él. Los modelos dinámicos de ajuste parcial encajan en este planteamiento. Establecen que los cambios en el porcentaje de establecimientos franquiciados $\left(\right.$ PFDOS $_{\mathrm{it}}-$ PFDOS $\left._{\mathrm{it}-1}\right)$ absorben parcialmente la diferencia entre el porcentaje objetivo de establecimientos franquiciados para el periodo $t\left(P F D O S^{*}{ }_{i t}\right)$ y dicho porcentaje en el periodo $t-1\left(P F D O S_{\mathrm{it}-1}\right)$ :

$$
\left(\text { PFDOS }_{\mathrm{it}}-\text { PFDOS }_{\mathrm{it}-1}\right)=\alpha\left(\text { PFDOS }_{\mathrm{it}}-\text { PFDOS }_{\mathrm{it}-1}\right)
$$

donde el coeficiente $\alpha, \in[0,1]$, mide la velocidad de ajuste, y está inversamente relacionado con los costes del ajuste que impiden alcanzar la estructura deseada. Operando obtenemos:

$$
\operatorname{PFDOS}_{\mathrm{it}}=\alpha P F D O S_{\mathrm{it}}+(1-\alpha) P F D O S_{\mathrm{it}-1}
$$

Si los costes de ajuste son igual a cero, es decir, $\alpha=1, P F D O S_{\text {it }}=$ PFDOS $_{\text {it }}$, con lo que las cadenas de franquicia alcanzan automáticamente su proporción objetivo de establecimientos franquiciados. Si, por el contrario, $\alpha=0, P F D O S_{\mathrm{it}}=P F D O S_{\mathrm{it}-1}$, lo que implica que los costes de transacción o ajuste son tan altos que las cadenas nunca llegan a la proporción objetivo. El proceso de ajuste es un equilibrio entre los costes de ajuste hacia el nivel objetivo de establecimientos franquiciados y los costes de no estar en equilibrio.

Dado que el nivel deseado de establecimientos franquiciados es inobservable, consideramos que puede se modelado como una función lineal de la escasez de recursos financieros y de los sistemas de incentivos de los franquiciadores. De esta manera, obtenemos la siguiente ecuación:

$$
\begin{aligned}
& \text { PFDOS }_{\text {it }}=a_{0}+a_{1} \text { INVINI }_{\text {it }}+a_{2} \text { END }_{\text {it }}+a_{3} \text { LIQUID }_{\text {it }}+ \\
& +a_{4} R e_{\text {it }}+a_{5} A N T G_{\mathrm{it}}+a_{6} E X T J_{\mathrm{it}}+a_{7} P M I N_{\mathrm{it}}+\mu_{\mathrm{it}}
\end{aligned}
$$

donde $P F D O S{ }^{*}$ it es el nivel objetivo de establecimientos franquiciados de una cadena $i$ en el año $t$ y sus variables explicativas son la inversión inicial 
(INVINI), el ratio de endeudamiento (END), el ratio de liquidez (LIQUID), la rentabilidad económica $(R e)$, la antigüedad de la cadena $(A N T G)$, los establecimientos en el extranjero (EXTJ) y la población mínima (PMIN).

$$
\text { Incorporando la ecuación (3) en la ecuación (2) y }
$$
considerando que los estimadores fueron calculados con datos de panel, obtenemos:

$$
\begin{aligned}
\text { PFDOS }_{\mathrm{it}}= & \alpha a_{0}+(1-\alpha) \text { PFDOS }_{\mathrm{it}-1}+\alpha a_{1} \text { INVINI }_{\mathrm{it}}+\alpha a_{2} E N D_{\mathrm{it}}+ \\
& +\alpha a_{3} \text { IQUID }_{\mathrm{it}}+\alpha a_{4} \operatorname{Re}_{\mathrm{it}}+\alpha a_{5} A N T G_{\mathrm{it}}+\alpha a_{6} \text { EXTJ }_{\mathrm{it}}+ \\
& +\alpha a_{7} \text { PMIN }_{\mathrm{it}}+\sum_{t=1996}^{2002} Y_{\mathrm{t}}+\gamma_{\mathrm{i}}+\mu_{\mathrm{it}}
\end{aligned}
$$

donde $\Sigma Y_{\mathrm{t}}$ es un conjunto de variables temporales dummy para cada año que recogen cualquier efecto temporal invariante de la empresa no incluido en la regresión. También incluimos $\gamma_{i}$, que es el efecto empresa, el cual asumimos constante para la empresa $i$ a lo largo de $t$; y $\mu_{\mathrm{it}}$, que es el término de error.

En las estimaciones, aplicamos el método generalizado de momentos (GMM) desarrollado para los modelos dinámicos de datos de panel por Arellano y Bond (1991). Esta metodología está específicamente diseñada para responder a dificultades econométricas relevantes en este trabajo: (a) la presencia de efectos individuales inobservables (en este caso, efectos de empresa). Los efectos individuales son eliminados tomando primeras diferencias de todas las variables; (b) el proceso autorregresivo en los datos relativos al comportamiento del nivel objetivo de establecimientos franquiciados (es decir, la necesidad de usar un modelo con variables dependientes retardadas para capturar la naturaleza dinámica de las decisiones sobre la estructura de propiedad de las cadenas); y (c) la posible endogeneidad de las variables independientes. El estimador de panel controla esta posible endogeneidad utilizando instrumentos internos, es decir, instrumentos basados en valores retardados de las variables explicativas.

La consistencia del estimador GMM depende de la validez de los instrumentos. Para afrontar esta cuestión, consideramos dos tests sugeridos por Arellano y Bond (1991). El primero es el test de sobreidentificación de las restricciones de Sargan, que prueba la validez conjunta de los instrumentos en la estimación del GMM. Esta prueba 
confirma la ausencia de correlación entre los instrumentos y el término de error de nuestro modelo. El segundo test examina la hipótesis de ausencia de correlación serial en los residuos de primera diferencia $\left(m_{2}\right)$. En nuestros modelos, esta hipótesis es siempre rechazada. Aunque existe una correlación serial de primer orden $\left(m_{1}\right)$ en los residuos diferenciados, es debido a la primera diferencia de los modelos.

\section{Variables}

La variable dependiente es definida como el porcentaje de establecimientos franquiciados sobre el total de establecimientos. Las variables independientes por las que se aproxima la escasez de capital por parte de la empresa se describen a continuación. La Tabla 2 muestra los estadísticos descriptivos y la Tabla 3 la correlación entre las variables.

a) Inversión inicial en el establecimiento (INVINI), definida como el importe, en millones de euros, que el franquiciado debe realizar para poner en marcha el negocio, incluyendo el canon de entrada. Pensamos que la inversión inicial nos da una idea de los recursos necesarios para la expansión de la cadena. Anticipamos que cuanto más alta sea dicha inversión, más recursos necesitará el franquiciador para afrontar la expansión de su cadena y, por tanto, recurrirá con una mayor probabilidad a los franquiciados como mecanismo de financiación ${ }^{16}$.

b) Ratio de endeudamiento (END), definido como ((Total Pasivo - Fondos Propios) / Total Pasivo) x 100. Refleja el peso de las deudas a corto y largo plazo sobre el total del pasivo de la empresa ${ }^{17}$. A medida que aumenta el nivel de endeudamiento de una empresa, su capacidad para obtener más recursos para financiar la expansión se reduce, en cuyo caso parece lógico pensar que pueda acudir a la franquicia, puesto que el franquiciado aporta buena parte de la inversión necesaria.

c) Ratio de liquidez (LIQUID), definido como (Activo circulante - Existencias) / Pasivo líquido. Está inversamente relacionado con el endeudamiento. Parece lógico pensar que cuando las empresas gozan de recursos disponibles para la inversión, una de las primeras

16 Esta variable ha sido utilizada por numerosos autores como Brickley y Dark (1987), Brickley, Dark y Weisbach (1991), Thompson (1992), Lafontaine (1992), Martin y Justis (1993) o López y Ventura (2002).

17 Norton (1995) o Combs y Ketchen (1999) han utilizado esta variable. 
opciones es reinvertirlo en la propia empresa. Esperaríamos, por tanto, una relación negativa con la propensión a franquiciar.

d) Rentabilidad económica (Re), definida como Resultado de explotación / Total activo. Indica el rendimiento que obtiene una empresa como consecuencia de sus inversiones en activos, con independencia de la estructura financiera de la misma ${ }^{18}$. Consideramos que cuanto mayor es la rentabilidad económica menor va a ser la propensión franquiciadora de las cadenas, puesto que cuanto mayor sea la rentabilidad obtenida en las inversiones propias, mayor será el interés de la empresa en acudir a otras fuentes de financiación diferentes a la franquicia ${ }^{19}$.

e) La variable $A N T G$ recoge el número de años que llevan las diferentes cadenas franquiciando en España ${ }^{20}$. Se supone que cuanta menor sea la experiencia acumulada por parte del franquiciador en su negocio más difícil le resultará obtener financiación, con lo que recurrirá a los franquiciados para poder acceder a la misma.

\section{Variables de control}

Hemos introducido dos variables de control para controlar los efectos del argumento de agencia. De esta manera, introducimos en el modelo:

f) Establecimientos en el extranjero $(E X T J)$, que mide el esfuerzo y dificultad para controlar al responsable del establecimiento. La variable EXTJ es una variable dummy que toma el valor 1 en aquellos casos en los que las cadenas posean establecimientos en el extranjero y 0 en el caso contrario. Puesto que la franquicia abarata el control de un conjunto de unidades dispersas, serán las cadenas que posean establecimientos fuera del territorio español las que tendrán una mayor proporción de establecimientos franquiciados. La razón es que, al tener una red más extensa y compleja, necesitan acudir más a los franquiciados ante la

18 Combs y Ketchen (1999) emplean, entre otras, esta variable para contratar el argumento financiero.

19 Como, por ejemplo, el endeudamiento o la ampliación de capital.

20 Esta misma variable ha sido empleada por Caves y Murphy (1976), Lafontaine y Kaufmann (1994), Minkler y Park (1994), Combs y Castrogiovanni (1994), López y Ventura (2002), Dant y Kaufmann (2003) o Castrogiovanni, Combs y Justis (2006). 
imposibilidad de supervisar directamente a los responsables de dichos establecimientos ${ }^{21}$.

g) Población mínima (PMIN), que mide la importancia del esfuerzo del responsable en el éxito del establecimiento. Recoge la población mínima exigida por la cadena, expresada en millones, que debe tener un determinado núcleo urbano para que un franquiciado pueda ubicar su establecimiento en dicha zona. Serán las cadenas que ofrecen productos o servicios más especializados las que exigirán una mayor población mínima, puesto que la demanda de los mismos es escasa y tienen que amortizar la inversión realizada. Por tanto, un porcentaje pequeño de la población está interesado en el producto, con lo que cada cliente es muy importante para el negocio y el responsable del establecimiento tiene que realizar un mayor esfuerzo en atraerlo y retenerlo.

Tabla 2: Estadísticos descriptivos

\begin{tabular}{|l|c|c|c|c|c|}
\hline Variable & Media & Desv. Std. & Mín. & Máx. & N \\
\hline PFDOS & 0,642 & 0,307 & 0,000 & 1,000 & 2765 \\
\hline INVINI & 0,079 & 0,104 & 0,000 & 1,683 & 3138 \\
\hline END & 82,305 & 59,354 & 0,000 & 993,220 & 3782 \\
\hline LIQUID & 4,019 & 98,439 & $-2,230$ & 5267,020 & 3747 \\
\hline Re & $-0,035$ & 1,092 & $-33,784$ & 10,318 & 3792 \\
\hline ANTG & 5,304 & 6,657 & 0,000 & 84,000 & 5469 \\
\hline EXTJ & 11,522 & 134,729 & 0,000 & 6000,000 & 2706 \\
\hline PMIN & 0,058 & 0,065 & 0,000 & 0,750 & 2134 \\
\hline
\end{tabular}

Tabla 3: Correlaciones

\begin{tabular}{|l|c|c|c|c|c|c|c|c|}
\hline & PFDOS & INVINI & END & LIQUID & Re & ANTG & EXTJ & PMIN \\
\hline PFDOS & 1,00000 & & & & & & & \\
\hline INVINI & $-0,1131$ & 1,00000 & & & & & & \\
\hline END & 0,0057 & $-0,0836$ & 1,00000 & & & & & \\
\hline LIQUID & $-0,0356$ & $-0,0306$ & $-0,1741$ & 1,00000 & & & & \\
\hline Re & 0,1273 & 0,0450 & $-0,1546$ & $-0,1209$ & 1,00000 & & & \\
\hline ANTG & 0,1594 & 0,0507 & $-0,1540$ & 0,0542 & 0,0805 & 1,00000 & & \\
\hline EXTJ & 0,0485 & $-0,0074$ & $-0,0490$ & 0,0035 & 0,0217 & 0,0487 & 1,00000 & \\
\hline PMIN & $-0,1043$ & 0,3060 & $-0,0090$ & $-0,0139$ & 0,0265 & $-0,0277$ & $-0,0351$ & 1,00000 \\
\hline
\end{tabular}

21 Lafontaine (1992) utiliza también esta variable como proxy del coste de supervisión. 


\section{RESULTADOS}

La Tabla 4 recoge los resultados del modelo econométrico, obtenidos con el programa Stata 8.0. En la primera columna hemos considerado como medida de los aspectos financieros, la inversión inicial, el endeudamiento y la antigüedad. En la segunda columna hemos considerado la liquidez de la empresa en lugar del endeudamiento dada la relación que presentan entre ellas. Y en la tercera columna hemos considerado la rentabilidad económica por el mismo motivo. Paralelamente, en todos los casos hemos metido como variables de control la población mínima y la presencia de establecimientos en el extranjero. Además, hemos considerado un grupo de variables dummies por años, que intentan recoger los efectos temporales. El método de estimación utiliza una serie de instrumentos, retardos en las variables, para resolver el problema de la endogeneidad de algunas variables. En nuestro caso, consideramos que pueden ser variables endógenas el endeudamiento, el ratio de liquidez, la rentabilidad económica y la presencia de establecimientos extranjeros, dejando como estrictamente exógenas la antigüedad de la cadena y la inversión inicial requerida por el establecimiento. Los coeficientes de estos instrumentos no se presentan, ya sea por convención de la propia metodología o por simplificar la tabla.

Tabla 4: Factores determinantes de la propensión franquiciadora

\begin{tabular}{|c|c|c|c|c|c|c|}
\hline \multirow{2}{*}{\begin{tabular}{|l|} 
Variable \\
PFDOS t-1
\end{tabular}} & \multicolumn{2}{|c|}{ Modelo 1} & \multicolumn{2}{|c|}{ Modelo 2} & \multicolumn{2}{|c|}{ Modelo 3} \\
\hline & $0,2190^{* * *}$ & $(5,90)$ & $0,3116^{* * *}$ & $(8.73)$ & $0.2639^{* * *}$ & $(8.47)$ \\
\hline INVINI & 0,0738 & $(0,71)$ & $-0,0312$ & $(-0.33)$ & 0.0408 & $(-2.90)$ \\
\hline END & $0,0003 * *$ & $(2,18)$ & & & & \\
\hline LIQUID & & & $-0,0044 * *$ & $(-2.28)$ & & \\
\hline $\operatorname{Re}$ & & & & & $-0.0287^{*}$ & $(-1.78)$ \\
\hline ANTG & $0,204^{* *}$ & $(1,96)$ & 0,0072 & $(1.53)$ & 0.0050 & $(1.37)$ \\
\hline EXTJ & 0,0000 & $(0,01)$ & 0,0002 & $(1.31)$ & $-0.0002 *$ & $(-1.70)$ \\
\hline PMIN & $-0,5740 * * *$ & $(-2,62)$ & $-0,2810 * *$ & $(-1.81)$ & -0.2630 & $(-1.12)$ \\
\hline dy98 & $0,0097 * * *$ & $(3,16)$ & $0,0103 * *$ & $(2.47)$ & $0.0126^{* * *}$ & $(4.54)$ \\
\hline dy00 & $-0,0113^{*}$ & $(-1,81)$ & $-0,0124 *$ & $(-1.94)$ & -0.0042 & $(-0.75)$ \\
\hline dy01 & $-0,0389 * * *$ & $(-4,01)$ & $-0,0429 * * *$ & $(-4.30)$ & $-0.0334 * * *$ & $(-3.55)$ \\
\hline dy02 & $-0,0574 * * *$ & $(-4,14)$ & $-0,0547 * * *$ & $(-3.69)$ & $-0.0453 * * *$ & $(-4.08)$ \\
\hline $\mathrm{m}_{1}$ & \multicolumn{2}{|c|}{$-1,69^{*}$} & \multicolumn{2}{|c|}{$-1,79 *$} & \multicolumn{2}{|c|}{$-1,74^{*}$} \\
\hline $\mathrm{m}_{2}$ & \multicolumn{2}{|c|}{$-1,30$} & \multicolumn{2}{|c|}{$-1,50$} & \multicolumn{2}{|c|}{$-1,12$} \\
\hline Sargan Test & \multicolumn{2}{|c|}{45,77} & \multicolumn{2}{|c|}{45,82} & \multicolumn{2}{|c|}{42,34} \\
\hline Observaciones & \multicolumn{2}{|c|}{322} & \multicolumn{2}{|c|}{322} & \multicolumn{2}{|c|}{325} \\
\hline Cadenas & \multicolumn{2}{|c|}{156} & \multicolumn{2}{|c|}{157} & \multicolumn{2}{|c|}{158} \\
\hline
\end{tabular}

Nota: Estadístico $t$ entre paréntesis $* * *, * *, *=$ Significativo al 99\%, 95\% y $90 \%$ respectivamente. 
En primer lugar, destaca observar que tanto los parámetros del endeudamiento, en la primera columna, como del ratio de liquidez, en la segunda, como de la rentabilidad económica, en la tercera, son significativos y con el signo esperado, tal y como predecía el argumento financiero. Esto es un claro soporte a dicho argumento financiero. Cuanto mayor es la liquidez y la rentabilidad económica y menor el endeudamiento menor es el uso de la franquicia. Esto deja abierta una pregunta. ¿Por qué es mejor la financiación a través de franquiciados que con capitales propios? Quizá una respuesta sea la menor incertidumbre que provoca al franquiciado poder dirigir personalmente su propio negocio. En el caso de convertirse en accionista debe soportar unos costes de agencia importantes.

En segundo lugar, sorprende la falta de significación de la inversión inicial y el signo contrario de la experiencia. Por lo que respecta a la variable de inversión inicial, conviene señalar que ha sido empleada en muchos otros trabajos previos obteniéndose para la misma unos resultados muy dispares ${ }^{22}$. Una probable explicación es que se produce un doble efecto contrapuesto. Por un lado, está el problema de la financiación, que llevaría a los franquiciadores a intentar franquiciar parte de sus establecimientos, pero, en sentido contrario, está la dificultad de encontrar franquiciados. El motivo es que, cuanto mayor sea el valor de la inversión inicial, mayor será el riesgo al que tiene que hacer frente el responsable del establecimiento y, en consecuencia, más tendencia existirá por parte del franquiciador a integrar sus puntos de venta puesto que, de esta manera, se asigna la variabilidad del riesgo a la parte que menos coste le supone asumirlo: el franquiciador ${ }^{23}$.

Por su parte, la experiencia es también significativa, pero con el signo contrario, positivo. Esto indica que a más experiencia más establecimientos franquiciados. Este es un resultado que no se puede argumentar por la falta de recursos. Todo lo contrario. Quizá pueda ser que

22 Así, por ejemplo, mientras autores como Brickley y Dark (1987), Brickley, Dark y Weisbach (1991), Lafontaine (1992) o López y Ventura (2002) obtienen que cuanto mayor es la inversión inicial menor es la propensión a franquiciar, lo que va en contra del argumento financiero, otros como Thompson (1992) y Martin y Justis (1993), obtienen el resultado contrario.

23 El franquiciado es más averso al riesgo que el franquiciador, puesto que, mientras el primero suele ser habitualmente un empresario individual cuyo patrimonio e ingresos suelen estar ligados a los resultados de su propio establecimiento, el segundo es normalmente una sociedad de mayor tamaño que suele contar con algún otro socio financiero, incluso con empresas de capital riesgo. Autores como Brickley y Dark (1987), Brickley, Dark y Weisbach (1991), Lafontaine (1992, 1993, 1995) o Perales y Vázquez (2003) respaldan este argumento. 
las empresas van desarrollando su modelo de negocio y van aprendiendo, con lo que cada vez se fían más del mismo. Es, por tanto, lógico pensar que si un franquiciador ha desarrollado una importante red durante varios años, su imagen entre los potenciales franquiciados sea buena y que muchos estén dispuestos a incorporarse a esta red como tales: saben que se introducen en un negocio probado extensamente y que el franquiciador tiene mucho capital reputacional que perder en caso de incumplimiento. El resultado esperado, en este caso, sería una relación positiva como la que hemos obtenido. Este resultado está en contraposición con la tendencia general que se observa en las variables temporales, significativas en su mayoría, a reducir la tendencia a la franquicia. Tanto la significación como los coeficientes de las variables temporales indican que, en relación a 1999, año de referencia, el porcentaje de establecimientos franquiciados es cada vez menor. Estos resultados encajan con la tendencia general observada por Pénard, Raynaud y Saussier (2003) y Lafontaine y Shaw (2005).

Finalmente, observamos que se verifica el modelo de ajuste parcial de la estructura de propiedad. El coeficiente de la variable dependiente retardada es muy significativo. Esto nos da una velocidad de ajuste entre un $78-69 \%$. Es una velocidad de ajuste alta, como era de suponer, puesto que los franquiciadores tienen a su disposición buenas herramientas para ajustarla. Sin embargo, hay que darse cuenta que no siempre encuentran a los franquiciados que desean y menos en las ciudades que ellos quieren. Esto puede obligarles a abrir con establecimientos propios para no perder su posición competitiva o para ocupar un buen local disponible, lo que no siempre es fácil encontrar en el centro de las grandes ciudades.

Por lo que respecta a las variables de control, los resultados obtenidos son dispares. Mientras que la variable EXTJ, encargada de medir los costes de controlar al responsable del establecimiento, presenta el signo positivo esperado, aunque sin ser significativo, en los dos primeros modelos, la variable $P M I N$ presenta un signo negativo, en todos los modelos, y significativo, en los dos primeros, lo cual es contrario a nuestras predicciones.

\section{CONCLUSIONES}

Este trabajo tiene como objetivo analizar si la dificultad para acceder a determinados recursos escasos, más concretamente a los 
financieros, es un factor determinante de la propensión franquiciadora de las cadenas españolas. Para ello, se ha tomado como muestra un panel de datos en el cual se recoge la información relativa a las cadenas de franquicia que se encontraban operando en nuestro país dentro del periodo 1996-2002. La ventaja de esta metodología radica en que nos permite controlar la heterogeneidad inobservable y recoger los efectos individuales de cada cadena, cuestión que no es recogida con las regresiones de corte transversal. Además, empleamos un modelo dinámico de ajuste parcial de la estructura de propiedad de las cadenas utilizando el método generalizado de los momentos en primeras diferencias, lo que nos permite, no sólo introducir un componente autorregresivo, sino también el empleo de variables explicativas endógenas.

Los resultados obtenidos muestran claramente que cuanto mayor es la liquidez y la rentabilidad económica y menor es el endeudamiento de una determinada cadena menor es su propensión a franquiciar establecimientos, lo que apoya el argumento de escasez de recursos financieros. Por el contrario, la variable de inversión inicial no es significativa y la de antigüedad de la cadena presenta un signo contrario al previsto. Lo primero puede ser explicado por la existencia de un doble efecto contrapuesto: por una parte, cuanto mayor es la inversión inicial más dificultades tiene el franquiciador para acceder a los recursos necesarios para ampliar su negocio, con lo que recurriría a los franquiciados como mecanismo de financiación, pero, por otra, mayor sería el riesgo al que tienen que hacer frente los potenciales franquiciados, los cuales son más aversos al riesgo que el franquiciador, con lo que este último optaría por mantener los establecimientos bajo su propiedad. Por lo que respecta a la variable de antigüedad, puede ocurrir que, más que medir la facilidad/dificultad de acceso a los recursos financieros, esté recogiendo el resultado del esfuerzo del franquiciador, en el sentido de que, al haber desarrollado su negocio durante varios años, ha conseguido crearse una buena imagen, con lo que resultará más fácil atraer a nuevos y buenos franquiciados, puesto que estos últimos saben que van a introducirse en un negocio rentable.

Finalmente, observamos que se verifica el modelo de ajuste parcial de la estructura de propiedad, siendo el coeficiente de la variable dependiente retardada muy significativo. Esto nos da una velocidad de ajuste muy alta, entre un $78-69 \%$, algo que era de esperar, puesto que los franquiciadores disponen de buenas herramientas para ajustarla. 


\section{BIBLIOGRAFÍA}

ARellano, M. and S. Bond (1991) "Some Tests of Specification for Panel Data: Monte Carlo Evidence and an Application to Employment Equations", Review of Economic Studies, Vol. 58, pp. 277-297.

BlaiR, R. and F. LAFONTAINE (2005) The Economics of Franchising. Cambridge: Cambridge University Press.

BRADACH, J. (1997) "Using the Plural Form in the Management of Restaurant Chains", Administrative Science Quarterly, Vol. 42, pp. 276-303.

- and R. ECCLES (1989) "Price, Authority and Trust: From Ideal Types to Plural Forms", Annual Review of Sociology, Vol. 15, pp. 97-118.

BRICKLEY, J. and F. DARK (1987) "The Choice of Organizational Form: The Case of Franchising", Journal of Financial Economics, Vol. 18, pp. 401-420.

-; F. DARK and M. WeISBACH (1991) "An Agency Perspective on Franchising", Financial Management, Vol. 20, pp. 27-35.

Castrogiovanni, G.; J. COMBS and R. Justis (2006) "Resource Scarcity and Agency Theory Predictions Concerning the Continued Use of Franchising in Multi-Outlet Networks", Journal of Small Business Management, Vol. 44, pp. 27-44.

CAVES, R. and W. MURPHY (1976) "Franchising: Firms, Markets and Intangible Assets", Southern Economic Journal, Vol. 42, pp. 572-586.

COMBS, J. and G. CASTROGIOVANNI (1994) "Franchisor Strategy: A Proposed Model and Empirical Test of Franchise Versus Company Ownership", Journal of Small Business Management, Vol. 31, pp. 37-48.

- and D. KeTCHEN (1999) "Can Capital Scarcity Help Agency Theory Explain Franchising? Revisiting the Capital Scarcity Hypothesis", Academy of Management Journal, Vol. 42, pp. 196-207.

DANT, R. (1995) "Motivations for Franchising: Rhetoric versus Reality", International Small Business Journal, Vol. 14, pp. 10-32.

- and P. KaUfmanN (2003) "Structural and Strategic Dynamics in Franchising", Journal of Retailing, Vol. 79, pp. 63-75.

HUNT, S. (1973) "The Trend Towards Company-Operated Units in Franchise Chains", Journal of Retailing, Vol. 49, pp. 3-13.

JeNSEN, M.C. and W.H. MECKLING (1976) "Theory of the Firm: Managerial Behavior, Agency Cost and Ownership Structure", Journal of Financial Economics, Vol. 3, pp. 305-360. 
LAFONTAINE, F. (1992) "Agency Theory and Franchising: Some Empirical Results", Rand Journal of Economics, Vol. 23, pp. 263-283.

- (1993) "Contractual Arrangements as Signaling Devices: Evidence from Franchising", Journal of Law, Economics and Organization, Vol. 9, pp. 256-289.

- (1995) "Pricing Decisions in Franchised Chains: A Look at the Fast-Food Industry", Documento de trabajo 5247, NBER.

- and P. KAUfMANN (1994) "The Evolution of Ownership Patterns in Franchise Systems", Journal of Retailing, Vol. 70, pp. 97-113.

- and K. SHAW (2005) "Targeting Managerial Control: Evidence from Franchising", Rand Journal of Economics, Vol. 36, pp. 131-150.

- and M. SLADE (2001) "Incentive Contracting and the Franchise Decision". K. Chatterjee and W. SAmuelson (eds.) Advances in Business Applications of Game Theory. Boston: Kluwer Academic Press.

LóPEZ, B. y J. VENTURA (2001) "Grupos estratégicos en las franquicias españolas: Características básicas", Economía Industrial, Vol. 340, pp. 163-176.

- y - (2002) "Integración vertical y causas de aparición de la franquicia", Revista Europea de Dirección y Economía de la Empresa, Vol. 11, pp. 55-74.

MARTIN, R. and R. JUSTIS (1993) "Franchising, liquidity Constraints and Entry", Applied Economics, Vol. 25, pp. 1269-1277.

MCGUIRE, E. (1971) Franchised Distributors. New York: The Conference Board.

MICHAEL, S. (2002) "First Mover Advantage Through Franchising", Journal of Business Venturing, Vol. 17, pp. 1-20.

MINKLER, A. (1990) "An Empirical Analysis of a Firm's Decision to Franchise", Economics Letters, Vol. 34, pp. 77-82.

- and T. PARK (1994) "Asset Specificity and Vertical Integration", Review of Industrial Organization, Vol. 9, pp. 409-423.

NORTON, S. (1988) "An Empirical Look at Franchising as an Organizational Form", Journal of Business, Vol. 61, pp. 197-217.

- (1995) "Is Franchising a Capital Structure Issue?", Journal of Corporate Finance, Vol. 2, pp. 75-101.

OXENFELDT, A. and A. KeLLY (1969) "Will Successful Franchise Systems Ultimately Become Wholly-Owned Chains?", Journal of Retailing, Vol. 44, pp. 69-87. 
OZANNE, U. and S. HUNT (1971) The Economic Effects of Franchising. US Select Committee on Small Business. Washington, D.C.: Us Government Printing Office.

PÉNARD, T.; E. RAYNAUd and S. SAUSSIER (2003) "Dual Distribution and Royalty Rates in Franchised Chains: An Empirical Analysis Using French Data", Journal of Marketing Channels, Vol. 10, pp. 5-31.

Perales, N. y L. VÁzQuez (2003) "Determinantes de la intensidad franquiciadora: Un enfoque de agencia", Investigaciones Económicas, Vol. 27, pp. 151-172.

RUBIN, P. (1978) "The theory of the firm and the structure of the franchise contract", Journal of Law and Economics, Vol. 21, pp. 223-233.

SEN, K. (1993) "The use of initial fees and royalties in business format franchising", Managerial and Decision Economics, Vol. 14, pp. 175-190.

SHANE, S. (1996) "Hybrid Organizational arrangements and their implications for firm growth and survival: A study of new franchisors", Academy of Management Journal, Vol. 39, pp. 316-334.

STANWORTH, J. and J. CURRAN (1999) "Colas, Burgers, Shakes and Shirkers: Towards a Sociological Model of Franchising in the Market Economy", Journal of Business Venturing, Vol. 14, pp. 323-344.

ThOMPSON, R. (1992) "Company Ownership versus Franchising: Issues and Evidence", Journal of Economic Studies, Vol. 19, pp. 31-42.

WILLIAMSON, O. (1991) "Comparative Economic Organization: The Analysis of Discrete Structural Alternatives", Administrative Science Quarterly, Vol. 36, pp. 269-296. 